\title{
Numerical approximation of basic reproduction number for an age-structured HIV infection model with both virus-to-cell and cell-to-cell transmissions
}

\author{
Kangkang Chang ${ }^{1}$ and zhang qimin ${ }^{2}$ \\ ${ }^{1}$ Ningxia University \\ ${ }^{2}$ School Mathematics and Computer Science, Ningxia University,
}

October 9, 2020

\begin{abstract}
In general, the basic reproduction number (R0) cannot be explicitly calculated for HIV(Human Immunodeficiency Virus) infection model with age-structured in a infinite dimensional spaces. To find R0, we need to transform the HIV model into a finite-dimensional space. In this paper, we are absorbed in numerical approximation of R0, which is the non-negative dominant eigenvalues of the positive irreducible matrices whose spectrum radius is defined as the next generation matrix. The linear operators generated by infected population are discretized into ordinary differential equations in a finite n-dimensional space. Thus, the abstract problem is transformed to find the positive dominant eigenvalues of the next generation matrix, we obtain a threshold R_0,n. Based on the spectral approximation theory, we show that $R_{-} 0, n-R 0$ as $n-+[?]$. Finally, by virtue of a numerical simulation, we demonstrate the results of the theorem.
\end{abstract}

\section{Hosted file}

one.pdf available at https://authorea.com/users/365748/articles/485726-numericalapproximation-of-basic-reproduction-number-for-an-age-structured-hiv-infection-modelwith-both-virus-to-cell-and-cell-to-cell-transmissions

\section{Hosted file}

one.tex available at https://authorea.com/users/365748/articles/485726-numericalapproximation-of-basic-reproduction-number-for-an-age-structured-hiv-infection-modelwith-both-virus-to-cell-and-cell-to-cell-transmissions 
figures/1181/1181-eps-converted-to.pdf 
figures/1182/1182-eps-converted-to.pdf 